\title{
Flavedo and albedo of five citrus fruits from Southern Italy: physicochemical characteristics and enzyme-assisted extraction of phenolic compounds
}

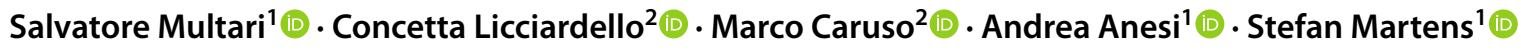

Received: 21 September 2020 / Accepted: 14 December 2020 / Published online: 3 January 2021

(c) The Author(s), under exclusive licence to Springer Science+Business Media, LLC part of Springer Nature 2021

\begin{abstract}
Peels are major byproducts of the juice processing industry. Citrus peels are composed of two tissues, namely the flavedo (outer layer) and the albedo (inner layer). Here, flavedo and albedo were obtained from the peel of grapefruit cv. 'Star ruby', lemon cv. 'Akragas', and sweet orange cvs. 'Tarocco', 'Sanguinello' and 'Moro'. Freeze dried flavedo and albedo were investigated for their functional properties, and subjected to enzyme-assisted extraction with pectinase and cellulase (concentration of $6 \mathrm{U} \mathrm{g}^{-1} \mathrm{DW}$ ) to extract the phenolic compounds. Albedo showed superior physicochemical characteristics than flavedo. Albedo from 'Tarocco' exhibited the maximum water holding capacity $(10.9 \pm 0.48 \mathrm{~g} / \mathrm{g})$. Grapefruit albedo revealed the highest water swelling $(13.4 \pm 2.62 \mathrm{~mL} / \mathrm{g})$ and oil binding $(6.31 \pm 2.62 \mathrm{~g} / \mathrm{g})$ capacities. As regards the enzyme-assisted extraction of phytochemicals, both enzymes enhanced the extraction of phenolics $(p<0.05)$ compared to the controls. In flavedo, pectinase and cellulase increased the recovery of phenolics by approx. 30\%, whereas in albedo the yields were increased above $60 \%$. Results from this investigation suggest that albedo might be used by the food industry as a novel raw material, due to its superior physicochemical properties. Moreover, results provide evidence that the enzyme-assisted extraction is advantageous to recover phenolics from the citrus peels.
\end{abstract}

\section{Graphic abstract}

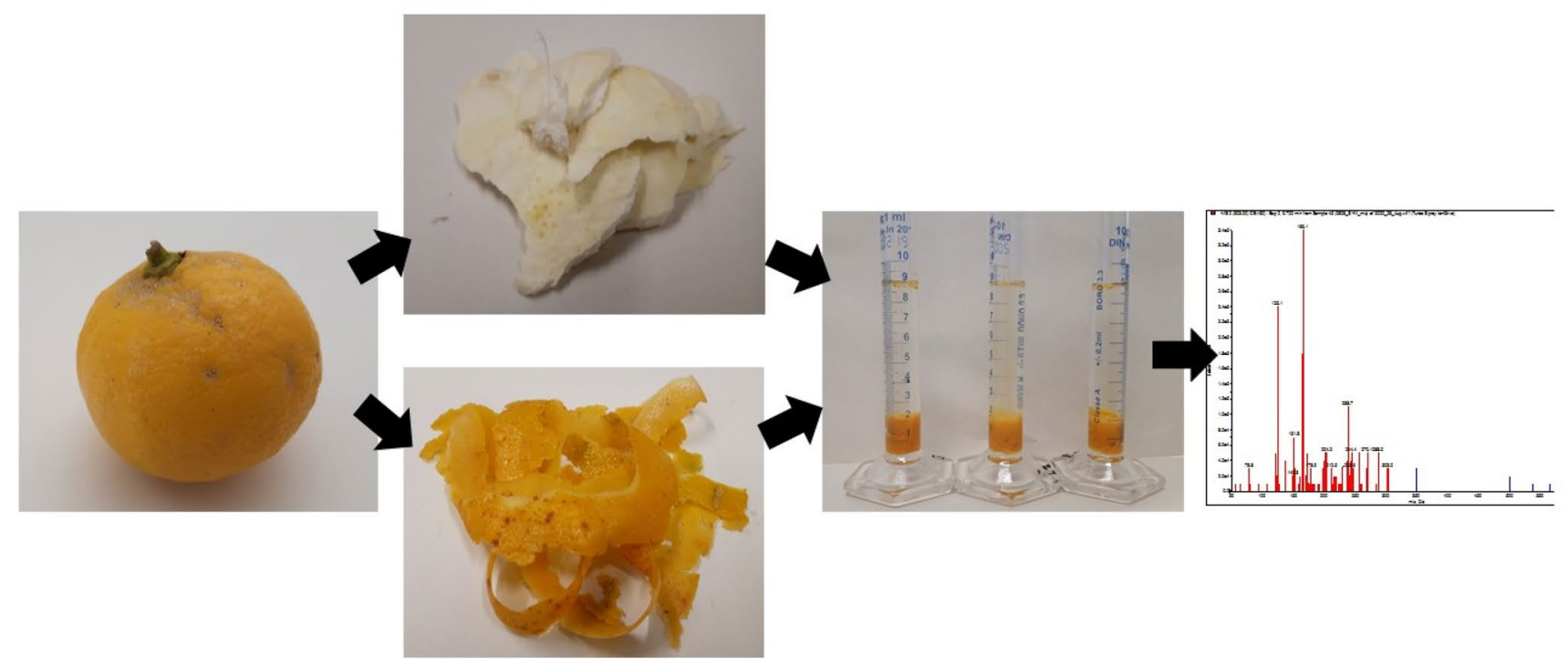

Supplementary Information The online version contains supplementary material available at https://doi.org/10.1007/s1169 4-020-00787-5.

Extended author information available on the last page of the article 
Keywords Cellulase $\cdot$ Pectinase $\cdot$ Fiber $\cdot$ Peel $\cdot$ Byproducts $\cdot$ Phytochemicals

$\begin{array}{ll}\text { Abbreviations } \\ \text { AE } & \text { Assisted extraction } \\ \text { BD } & \text { Bulk density } \\ \text { CTRL } & \text { Control } \\ \text { DW } & \text { Dry weight } \\ \text { GLCS } & \text { Glucoside } \\ \text { GrAl } & \text { Grapefruit albedo } \\ \text { GrFl } & \text { Grapefruit flavedo } \\ \text { LeFl } & \text { Lemon flavedo } \\ \text { LeAl } & \text { Lemon albedo } \\ \text { MoAl } & \text { Moro albedo } \\ \text { MoFl } & \text { Moro flavedo } \\ \text { OBC } & \text { Oil binding capacity } \\ \text { PCA } & \text { Principal component analysis } \\ \text { SD } & \text { Standard deviation } \\ \text { SaFl } & \text { Sanguinello flavedo } \\ \text { SaAl } & \text { Sanguinello albedo } \\ \text { TaFl } & \text { Tarocco flavedo } \\ \text { TaAl } & \text { Tarocco albedo } \\ \text { WHC } & \text { Water holding capacity } \\ \text { WSC } & \text { Water swelling capacity }\end{array}$

\section{Statement of novelty}

Citrus fruits are products of great market value, as used by the juice industry in huge quantities. The industry processes millions of tons of citrus fruits per year, but only the pulp is utilized, whereas peels are mostly discarded. This generates vast amounts of byproducts ( $>100$ million tons/year), since the peel can make up to $50 \%$ of the weight of the fresh fruit. This investigation showed that citrus peels comprise two tissues of technological interest, i.e., flavedo and albedo, which could find applications in the food sector. In addition, flavedo and albedo are prominent sources of phytochemicals, e.g., phenolic compounds. These compounds could be utilized by the food, cosmetics, and pharmaceutical industries. The recovery of the citrus peels would provide economic and environmental benefits. The study provides the food manufacturers with valuable information about the use of citrus by-products, and supports the comprehensive utilization of all the citrus tissues.

\section{Introduction}

The citrus processing industry generates vast amounts of byproducts, mainly in the form of peel that can make up to $50 \%$ of the fruit weight [1]. Citrus peels comprise two tissues, namely the flavedo (the outer colored layer) and the albedo (the white inner layer). As regards the chemical composition, flavedo and albedo are abundant in soluble and insoluble fiber, as well as lipids and organic acids [2]. Flavedo and albedo find applications in the production of jams, confectionary, and cosmetics, but their use is limited [3]. Italy is a major producer of citrus fruits within the $\mathrm{EU}$, and in the year 2018 produced about 3 million tons of citrus fruits [4], coming mostly from the Southern regions. Hence, it would be advantageous to recover the discarded tissues through integrated approaches.

It is recognized that dietary fiber ameliorate the physicochemical characteristics of numerous food products, for instance by strengthening the stability of gels and emulsions [5]. Fiber from citrus has demonstrated high water swelling and holding capacities, hence its incorporation in processed food would augment the viscosity of the systems [6]. Although several authors have investigated the functional characteristics of different citrus peels, bulk raw materials were employed and no distinction was performed between flavedo and albedo [7]. These two tissues, having different chemical composition, are likely to perform differently from the physicochemical standpoint.

It has been demonstrated that citrus peels are major sources of biological active phenolic compounds [8]. Citrus distinctive phytochemicals, such as the polymethoxylated flavones, e.g., tangeretin, diosmetin, and sinensetin, are found at different concentrations not only amongst the different citrus species $[9,10]$, but also across the tissues of the same fruit [11]. In citrus peels, as in other fibrous materials, phenolics are primarily bound to the cell wall polymers [12]. Enzymatic treatments have been successfully employed to disrupt the cell wall structure and enhance the extractability of the phenolic compounds [13, 14]. Nevertheless, as per the peel functionality, authors have investigated gross raw materials, missing to distinguish between flavedo and albedo, which for structural and environmental reasons exhibit different phenolic profiles [11].

In this study, the peels of five citrus fruits were analyzed, namely grapefruit cv. 'Star ruby', lemon cv. 'Akragas', and sweet orange cvs. 'Tarocco', 'Sanguinello', and 'Moro'. Peels were sectioned in flavedo and albedo. This study aimed to (1) investigate the functional (physicochemical) characteristics of flavedo and albedo from the 
selected fruits, (2) perform the enzyme-assisted extraction (AE) of phenolic compounds, and evaluate the effects of cellulase and pectinase application on the flavedo and albedo.

\section{Materials and methods}

\section{Chemicals}

General laboratory reagents were purchased from SigmaAldrich (Milan, Italy) and FisherScientific (Milan, Italy), and were of analytical grade. Acetonitrile and methanol were of LC-MS grade. Deionized water was purified in loco with Arium ${ }^{\circledR}$ purification system (Sartorius AG, Goettingen, Germany). Authentic standards of phenolic compounds were purchased from Sigma-Aldrich (Milan, Italy), TransMIT (Gießen, Germany), and Phytolab GmbH \& Co (Estenbergsgreuth, Germany). The $0.22 \mu \mathrm{m}$ PTFE membranes were purchased from Merck Millipore, Darmstadt, Germany. The enzymes cellulase (from Trichoderma ree-

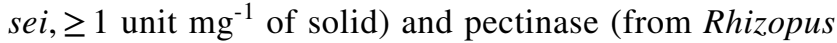
sp., 400-800 units $\mathrm{g}^{-1}$ of solid) were obtained from SigmaAldrich (Milan, Italy).

\section{Plant materials, and sample preparation}

Fruits from grapefruit (Citrus paradisi Macf.) cv. 'Star ruby', lemon [C. limon (L.) Burm. f] cv. Akragas, and pigmented sweet oranges [C. sinensis (L.) Osbeck] cvs. 'Tarocco', 'Sanguinello', and 'Moro', were cultivated at the CREA experimental fields of Palazzelli $\left(37^{\circ} 20^{\prime} 22^{\prime \prime}\right.$ $\left.\mathrm{N}, 14^{\circ} 53^{\prime} 31^{\prime \prime} \mathrm{E}\right)$ and Acireale $\left(37^{\circ} 36^{\prime} 31^{\prime \prime} \mathrm{N}, 15^{\circ} 09^{\prime} 56^{\prime \prime}\right.$ E), in Sicily, Southern Italy. Citrus fruits will be hereafter referred as GR (grapefruit), LE (lemon), TA ('Tarocco'), SA ('Sanguinello'), and MO ('Moro'). Three trees from each variety were chosen to carry out the sampling, with ten fruits being harvested from each tree (three biological replicates). Fruits were collected in November 2019. Then, fruits were washed, and the two tissues constituting the peel were separated, namely the flavedo and the albedo. Samples were freeze-dried, milled with a ball mill (CryoMill from Retsch GmbH, Haan, Germany), and stored under vacuum at room temperature in desiccators. Results were expressed as dry weight (dw).

\section{Functional characteristics}

The functional characteristics of the citrus tissues albedo and flavedo were assessed by adapting the methods described by Zhang et al. [15] and Oladapo Olukomaiya et al. [16]. The water holding capacity (WHC) and oil binding capacity
$(\mathrm{OBC})$ were measured using approx. $0.20 \mathrm{~g}$ of samples, and calculated with the following equation:

WHC and $\mathrm{OBC}\left(\frac{g}{g}\right)=\frac{(W 2-W 0)}{(W 1-W 0)}$

where $\mathrm{W}_{0}$ is the weight of the empty tube, $\mathrm{W}_{1}$ is the weight of the tube with the dry sample, and $\mathrm{W}_{2}$ is the weight of the tube with the hydrated (or containing the oil) sample.

The water swelling capacity (WSC) was calculated with the following equation:

$\operatorname{WSC}\left(\frac{\mathrm{mL}}{\mathrm{g}}\right)=\frac{(V 1-V 0)}{W 0}$

where $\mathrm{V}_{0}$ is the volume of the dry sample, $\mathrm{V}_{1}$ is the volume of the hydrated sample, and $\mathrm{W}_{0}$ is the weight of the dry sample.

The bulk density (BD) was measured by introducing approx. $1 \mathrm{~g}$ of dry sample in a $10 \mathrm{~mL}$ graduated cylinder. The cylinder was tapped several times. The $\mathrm{BD}(\mathrm{g} / \mathrm{mL})$ was expressed as weight of the sample (g) per sample volume $(\mathrm{mL})$.

\section{Enzyme-assisted extraction of phenolic compounds}

The enzyme-assisted extraction (AE) of phenolic compounds from flavedo and albedo was performed using the enzymes pectinase and cellulase, adapting the method from Ruviaro et al. [13]. The conditions of extraction were chosen after initial evaluation of the effects of single parameters, i.e., extraction temperature $\left(40,50\right.$, and $\left.60^{\circ} \mathrm{C}\right)$, enzyme concentration $\left(5,6,7 \mathrm{U} \mathrm{g}^{-1}\right)$, and extraction time $(6,12,24 \mathrm{~h})$. Each parameter was assessed three times, with extracts being analyzed through LC-MS/MS as described below.

Following evaluation of the preliminary data, the enzymatic reaction medium was prepared by dissolving the enzymes (final dosage $=6 \mathrm{U} \mathrm{per}^{-1}$ of citrus DW) in sodium acetate buffer $(20 \mathrm{mM}, \mathrm{pH} 5.0)$. Then, samples were mixed with the enzymatic medium, and stirred (400 rpm) overnight at $40{ }^{\circ} \mathrm{C}$. The treatment was stopped by heating the samples at $95{ }^{\circ} \mathrm{C}$ for $5 \mathrm{~min}$. Subsequently, samples were cooled in an ice bath, and $1 \mathrm{~mL}$ of ethanol was added. The phenolic compounds were extracted three times into $6 \mathrm{~mL}$ of ethyl acetate (stirred at RT for 10 min and sonicated in an ultrasonic bath for $5 \mathrm{~min}$ ). The organic layers were separated by centrifugation $\left(5 \mathrm{~min} ; 3000 \times g ; 18{ }^{\circ} \mathrm{C}\right)$, pooled, dried under reduced pressure at temperature not exceeding $40{ }^{\circ} \mathrm{C}$. Then the dry extracts were dissolved in $1 \mathrm{~mL}$ of LC-MS methanol. Control samples were treated under the same conditions but without the addition of the enzymes. Rosmarinic acid was used as the internal standard to a final concentration of $3 \mathrm{mg} \mathrm{L}^{-1}$. 
The identification and quantification of the phenolic compounds was performed through UPLC-MS/MS analysis as described by Multari et al. [17]. It was employed a Waters Acquity UPLC system (Milford, MA, USA) equipped with a binary pump, an online vacuum degasser, an autosampler, and a column compartment. The separation of the phenolic compounds was obtained on a Waters Acquity HSS T3 column $1.8 \mu \mathrm{m}, 100 \mathrm{~mm} \times 2.1 \mathrm{~mm}$, kept at $35{ }^{\circ} \mathrm{C}$. Data were processed using the Mass Lynx Target Lynx Application Manager (Waters), and multiple reaction monitoring (MRM) was used for quantification based on the peak area of the samples. Table S1 reports the MRM parameters.

\section{Statistical analysis}

Data reported are means of minimum three independent observations and values are expressed as mean \pm SD. Differences among groups were considered significant at $p<0.05$. PCA was applied to identify the components that contributed mostly to the variability amongst the samples. The PCA was performed on mean-centered data that were scaled by the standard deviation ("unit variance" scaling). Selected data were analyzed using one-way ANOVA followed by posthoc Tukey HSD test for multiple comparison. The statistical analysis was performed using PAST 3.24 (Oslo, Norway).
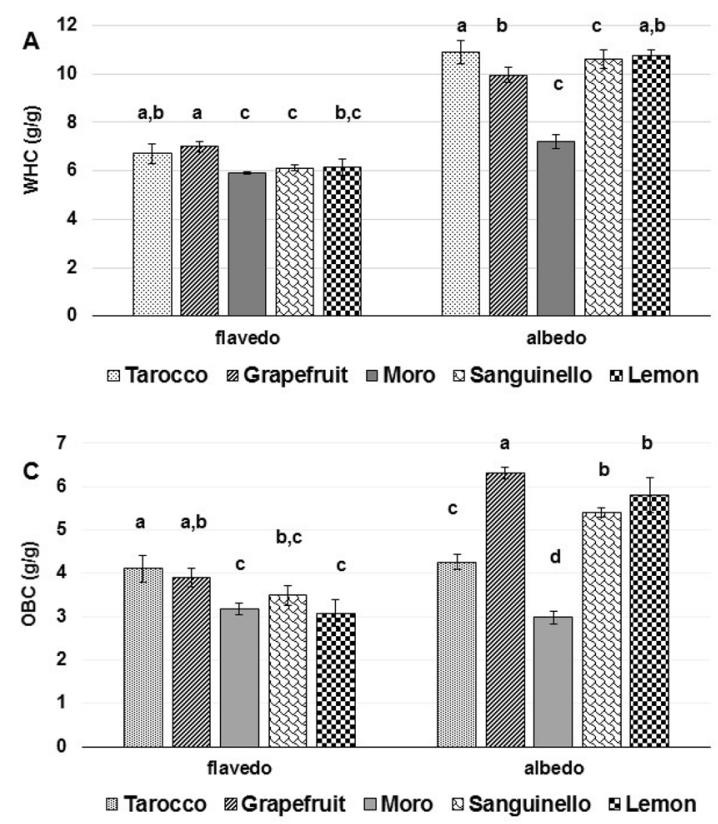

\section{Results and discussion}

\section{Functional characteristics}

Figure 1a-d show the functional characteristics of the selected citrus tissues. The WHC is expressed as $\mathrm{g}$ water/g citrus tissue. The WHC of flavedo ranged between $5.92 \pm 0.03(\mathrm{MO})$ and $6.99 \pm 0.22 \mathrm{~g} / \mathrm{g}(\mathrm{GR})$, whereas WHC of albedo ranged between $7.22 \pm 0.29(\mathrm{MO})$ and $10.9 \pm 0.48 \mathrm{~g} / \mathrm{g}$ (TA). Significant differences $(p<0.05)$ were observed amongst the samples. Data from the chosen fruits are consistent with a previous study performed on citrus peel fiber [18]. With regard to the type of tissue, albedo had on average 34\% higher WHC than flavedo. In both groups, 'Moro' exhibited the lowest WHC values. Since the WHC is mainly due to the presence of hydrophilic groups that bind molecules of water, it is likely that the matrices from 'Moro' contained lower amounts of hydrophilic functional groups. In particular, pectin is a soluble fiber formed of galacturonic acid residues, which has the greatest WHC amongst the dietary fiber $[19,20]$. It is possible that 'Moro' orange might contain lower amounts of pectin than the other citrus fruits. To the authors' knowledge, there are no previous studies that explored the dietary fiber composition of 'Moro'. Further research is needed to clarify the link between chemical composition, mostly monosaccharides, and WHC in citrus fruits.

The WSC, expressed as $\mathrm{mL}$ of water per $\mathrm{g}$ of sample, is attributable to the microstructure of the matrix. The WSC of flavedo ranged from $3.13 \pm 0.26$ (LE) to $6.68 \pm 0.64 \mathrm{~mL} \mathrm{~g}^{-1}$
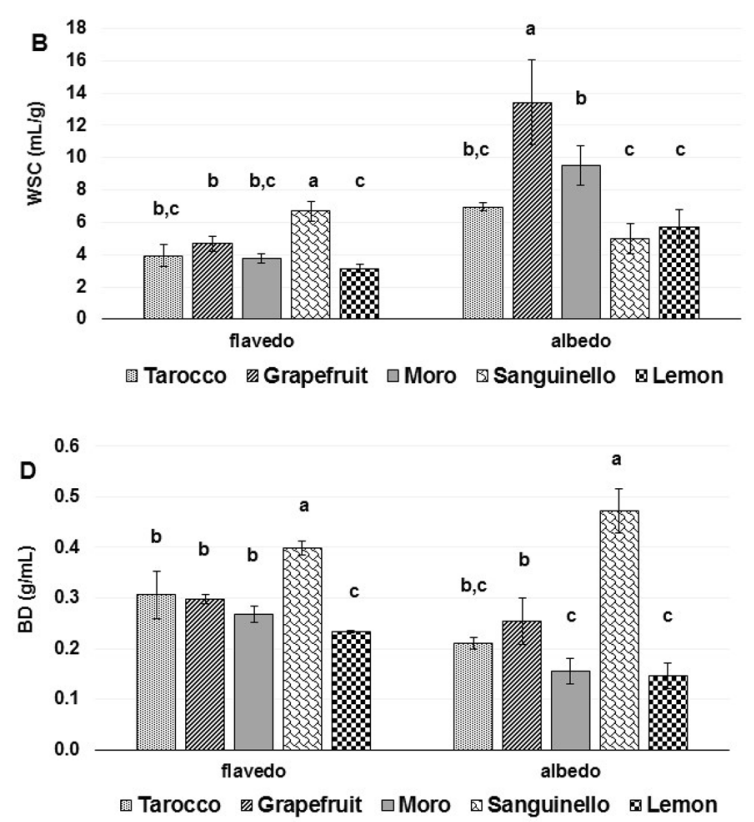

Fig. 1 Functional characteristics of citrus flavedo and albedo: a WHC, b WSC, $\mathbf{c}$ OBC, $\mathbf{d}$ BD. Values are expressed as mean \pm SD ( $\mathrm{n}=4)$. Values within a group with different letters are significantly different $(p \leq 0.05)$ 
(SA) $(p<0.05)$, whereas in the albedo it ranged from $5.0 \pm 0.92(\mathrm{SA})$ to $13.4 \pm 2.62 \mathrm{~mL} \mathrm{~g}^{-1}(\mathrm{GR})(p<0.05)$. The albedo performed considerably better than the flavedo as regards WSC, showing values about 35\% higher, in the same way as the WHC. This suggests that the two functional properties are regulated by similar factors. Sanguinello distinguished from the other fruits, since the flavedo outperformed the albedo $(+25 \%, p=0.024)$. Sanguinello flavedo might present a micro-structure that favors the WSC, e.g., high porosity, honey-comb organization, unsystematic fiber structure [15].

The OBC is expressed as $\mathrm{g}$ oil/ $\mathrm{g}$ citrus tissue and is presented in Fig. 1c. The OBC of flavedo varied between $3.08 \pm 0.31$ (LE) and $4.11 \pm 0.30 \mathrm{~g} / \mathrm{g}(\mathrm{TA})$, whereas in albedo varied between $2.99 \pm 0.14(\mathrm{MO})$ and $6.31 \pm 0.12 \mathrm{~g} / \mathrm{g}(\mathrm{TA})$. These results revealed that citrus albedo had higher OBC than flavedos. With reference to the type of matrix, the OBC values were significantly different $(p<0.05)$ within the same matrix group. The mechanism of oil adsorption is not completely established, however, the presence of cellulosic fiber and an elevated number of acyl groups might improve this characteristic [21]. Several authors have investigated the functional properties of citrus peels, however, bulk raw materials were employed and, up to now, a distinction between the two tissues constituting the peel was not performed. This might explain why samples from the present study showed higher OBC than those reported previously $[21,22]$.

The BD is expressed as $\mathrm{g} \mathrm{mL}^{-1}$ and is presented in Fig. 1d. In flavedo it ranged from $0.23 \pm 0.00$ (LE) to $0.40 \pm 0.01 \mathrm{~g} \mathrm{~mL}^{-1}(\mathrm{SA})$, whereas in albedo it ranged from $0.15 \pm 0.03$ (LE) to $0.47 \pm 0.44 \mathrm{~g} \mathrm{~mL}^{-1}$ (SA). Excepting SA, albedo showed lower BD than flavedo. Most likely, albedo being highly porous materials [23, 24] were made of less dense and lighter particles. On the contrary, flavedo being rich in oils [25] delivered particles that aggregated and formed bigger complexes. Regardless of the specific tissue, SA provided the fractions with the highest $\mathrm{BD}$, whereas $\mathrm{LE}$ tissues performed conversely. This indicates that the particles obtained from SA were the densest and the heaviest, in contrast, the tissues of LE delivered particles particularly light.

\section{Enzyme-assisted extraction of phenolic compounds}

Citrus flavedo and albedo were treated with pectinase and cellulase to enhance the release of phenolic compounds from the fibrous materials. In general, the enzymatic treatments improved $(p<0.05)$ the extraction efficiency of the phenolic metabolites (Table 1). In the flavedo group, the application of the enzymes increased the phenolic yield of approx. 30\%. 'Moro' provided the flavedo that benefited the most from the use of the enzymes, with concentrations of

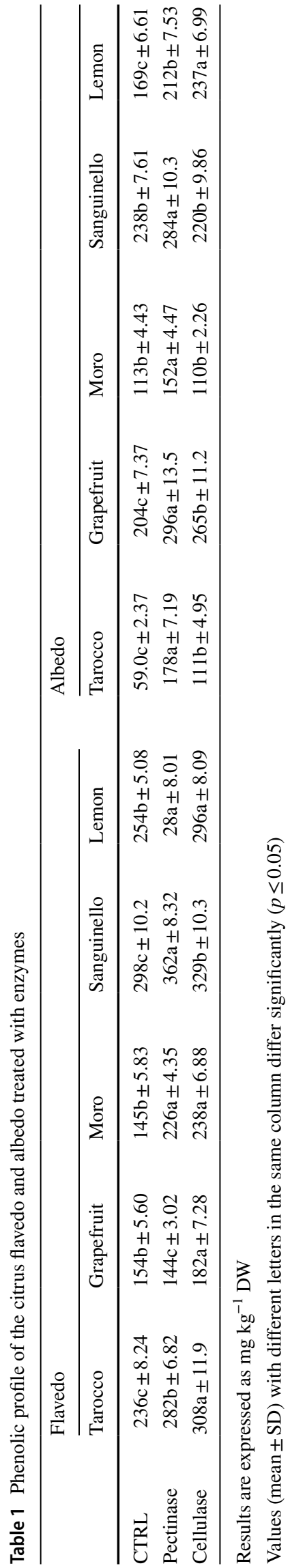


cumulative phenolics that increased from $145 \pm 5.83$ (ctrl) to $226 \pm 4.35$ (pectinase-AE) and $238 \pm 6.88$ (cellulase-AE) $\mathrm{mg} \mathrm{kg}^{-1}$. The treatments resulted particularly effective on the albedo, with yields of cumulative phenolics increasing above $60 \%$. 'Tarocco' provided the albedo most functional to the enzymatic hydrolysis, with concentration of cumulative phenolics growing from $59.0 \pm 2.37$ (ctrl) to $178 \pm 7.19$ (pectinase-AE.) and $111 \pm 4.95$ (cellulase-AE) $\mathrm{mg} \mathrm{kg}^{-1}$. On the whole, the application of enzymes prompted the digestion of the peel tissues, and subsequent extraction of the intracellular phenolics, with only few exceptions being observed, namely the GR flavedo was unaffected by pectinase $(p \geq 0.05)$, whereas MO and SA albedo were unaffected by cellulase ( $p \geq 0.05)$.

Hesperidin was the main phenolic compound found in the control of the citrus tissues (Table S2), peaking to $91.9 \pm 3.09 \mathrm{mg} \mathrm{kg}^{-1}$ in SA flavedo. Other investigations performed on citrus reported similar values $[11,15]$. Hesperidin was increased in the albedo group, e.g., $8.05 \pm 0.97 \mathrm{mg} \mathrm{kg}^{-1}$ (approx. 10-folds increase) in the GR albedo cellulasetreated. In the flavedo, the treatments led to irregular outcomes, with the compound decreasing in 'Tarocco' pectinase-treated. Hesperidin is a flavanone glycoside that is composed by the aglycone hesperetin attached to a rutinose disaccharide [26]. Since in 'Tarocco' flavedo the reduction in hesperidin was offset by an increase in hesperetin, it is very likely that the enzyme pectinase performed an efficient digestion of the tissue, which led to the hydrolysis of the flavanone glycoside with consequent release of the aglycones moiety. Narirutin is a flavanone glucoside abundant in orange peel [27] that increased $(p<0.05)$ when TA, MO, and SA were treated with pectinase. Pectin is the most abundant polysaccharide of orange albedo [28]. Since narirutin aggregates with pectin to form physical stable particles [29, 30], it is likely that the enzyme pectinase by digesting the pectin found in the albedo might have released narirutin to a great extent. Eriocitrin peaked in lemon, i.e., $35.4 \pm 1.14$ and $22.0 \pm 0.99 \mathrm{mg} \mathrm{kg}^{-1}$ in flavedo and albedo, respectively. Pectinase doubled the eriocitrin concentrations in both lemon flavedo and albedo, $71.7 \pm 0.16$ and $54.3 \pm 4.81 \mathrm{mg} \mathrm{kg}^{-1}$, respectively. It is clear that the ability of the enzymes to unloose the bound phenolic compounds depended largely on the sample composition, mostly the quality and quantity of fiber, and in some matrices the hydrolytic activity might have been very extensive, causing the degradation of the compounds. In terms of phenolic acids, the treatment with enzymes augmented significantly (Table S2; $p<0.05)$ their extraction yields. Amongst the hydroxycinnamates, ferulic acid resulted the most abundant, followed by $m$-coumaric and $p$-coumaric acids. 'Tarocco' provided the tissues most responsive to the enzymes. In particular, pectinase doubled the concentrations of ferulic acid in TaFl $\left(6.06 \pm 0.54 \mathrm{mg} \mathrm{kg}^{-1} ; p=0.0002\right)$, whereas in TaAl concentrations raised from $0.14 \pm 0.01$ (ctrl) to $6.54 \pm 0.53 \mathrm{mg} \mathrm{kg}^{-1}$ $(p=0.0001)$. The hydroxybenzoic acid derivatives were more affected by the treatment with cellulase, above all vanillic acid, which peaked in LeFl $\left(56.0 \pm 2.90 \mathrm{mg} \mathrm{kg}^{-1}\right.$; $p=0.0084)$ and $\mathrm{GrAl}\left(36.8 \pm 1.70 \mathrm{mg} \mathrm{kg}^{-1} ; p<0.0001\right)$. In general, the employed enzymes facilitated the release of the phenolic compounds, which being often attached to the pectin and cellulosic polysaccharides of the cell walls, require hydrolytic processes to be efficiently extracted [31,32].

PCA was applied to discriminate the samples on the basis of the genotype, the tissue, and the enzymatic treatment (Fig. 2). The data matrix comprised the phenolic metabolites detected (about 40) and listed in Table S2. The two first PCs explained $53.3 \%$ of the total variability, with $\mathrm{PC} 1$ accounting for $37.2 \%$ and PC2 for $16.1 \%$ of variability, respectively. The score plot displays a clustering of the samples based on the characteristic of genotype, with a marked separation of fruits from grapefruit and lemon that occupied the upper side of PC2. This indicates that the individual genotypes
Fig. 2 PCA Score Plot of citrus flavedo and albedo in function of PC1 and PC2. The considered phenolic compounds are those listed in Table S1. Symbol legend: dots ('Tarocco' sweet orange), squares (grapefruit), triangles ('Moro' sweet orange), diamonds ('Sanguinello' sweet orange), plus/cross (lemon). Color legend: filled symbols (pectinase treatment), empty symbols (cellulase treatment), black symbols (control). Further details on the PCA analysis are provided in Figs. S2-3

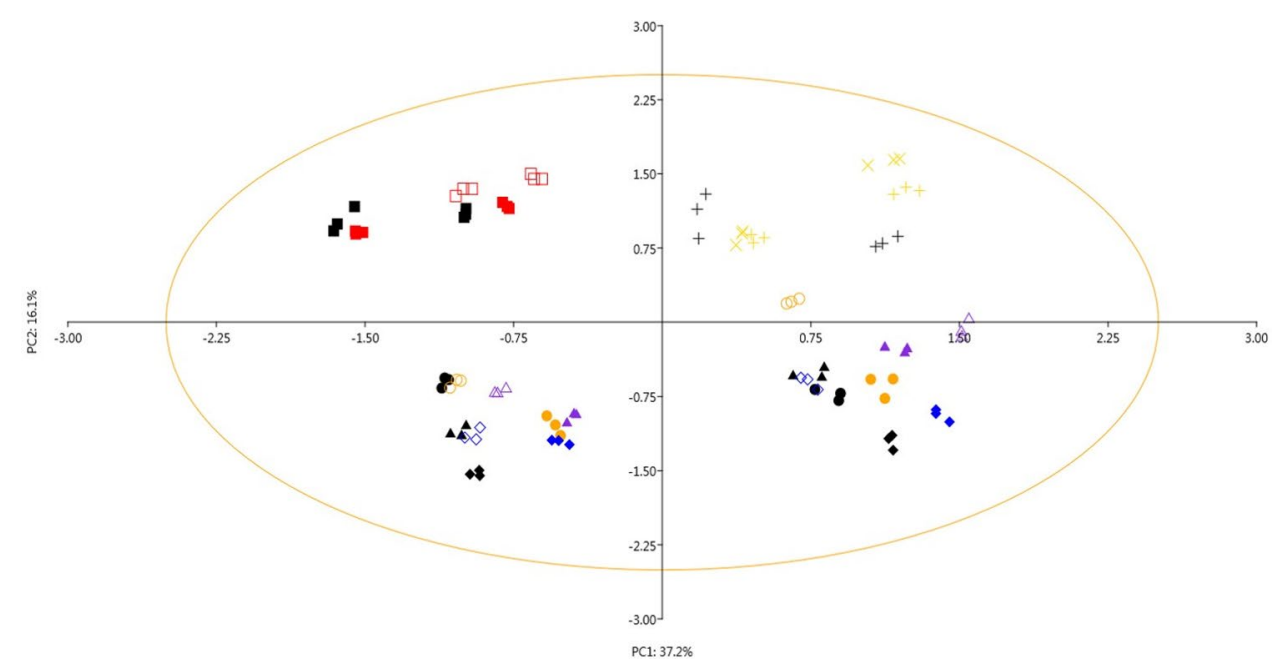


Fig. 3 PCA Biplot of citrus flavedos and albedos in function of PC1 and PC2. The considered phenolic compounds are those listed in Table S1. Symbol legend: dots ('Tarocco' sweet orange), squares (grapefruit), triangles ('Moro' sweet orange), diamonds ('Sanguinello' sweet orange), plus/cross (lemon). Color legend: filled symbols (pectinase treatment), empty symbols (cellulase treatment), black symbols (control). Further details on the PCA analysis are provided in Fig. S1

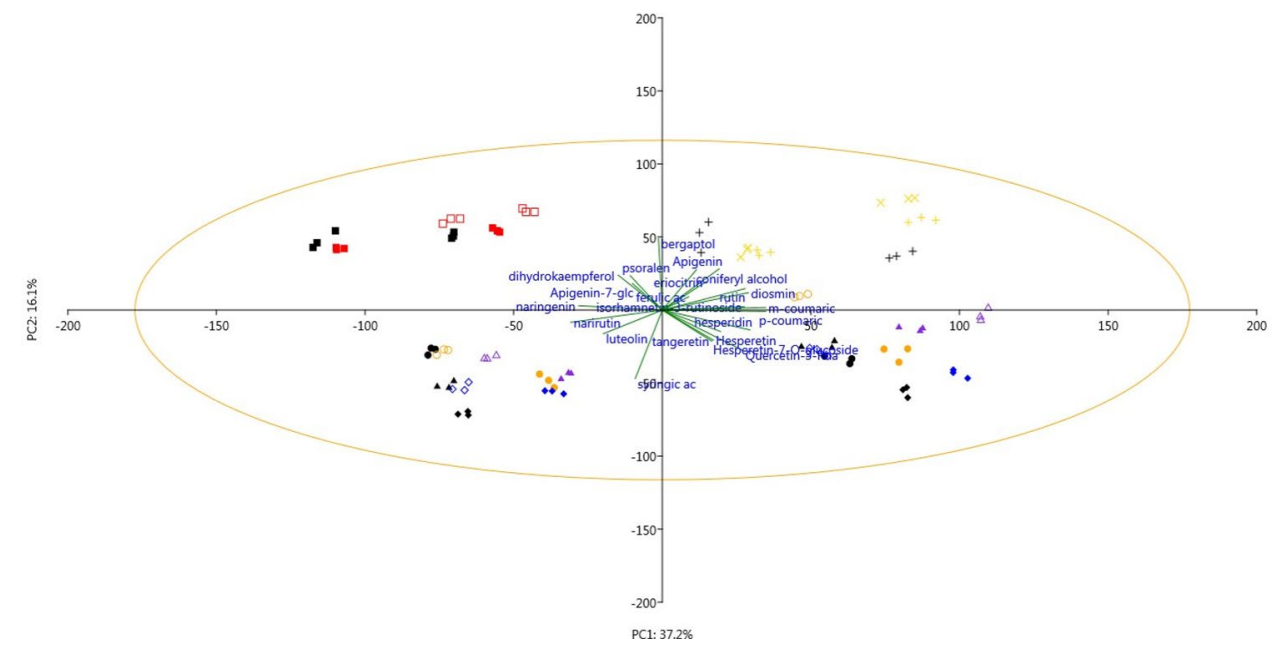

maintained their specific metabolomic characteristics even after the treatments. The biplot graph (Fig. 3) shows that the compounds dihydrokaempferol, apigenin 7-O-glucoside, and naringenin, contributed to distinguish the grapefruit samples, which provided sizeable amounts of these compounds. Phenolic metabolites, such as bergaptol, coniferyl alcohol, and rutin contributed to locate samples from lemon on the upper right-hand quarter. Samples from the sweet oranges (TA, SA, and MO) tended to group on the negative side of $\mathrm{PC} 2$. In these three genotypes, the tissue resulted the discriminating factor, since the flavedo samples occupied the right-hand quarter, whereas the albedo samples occupied the left-handed quarter (Fig. S1). The three varieties of orange belong to the same species, i.e., Citrus sinensis (L.) Osbeck. The PCA model showed that the three orange varieties maintained the metabolic features associated to the species, even after the enzymatic treatments. The metabolites that resulted distinctive of three orange varieties were tangeretin and hesperetin for the tissue flavedo, whereas luteolin and syringic acid were distinctive of the orange albedo (Fig. 3),

\section{Conclusions}

The present study demonstrated that the two tissues constituting the citrus peel, namely flavedo and albedo, have advantageous functional properties. In particular, the albedo showed pronounced water holding and swelling capacities. These are characteristics that are functional to form gels and increase the viscosity of the food systems, hence citrus albedo could find applications in the production of jams, yogurts, and emulsions. Amongst the selected species, tissues from grapefruit revealed the most prominent functional characteristics and the potential to be used as food ingredients. Grapefruit albedo could be used as a fat replacer to reformulate traditional high-calorie products, since the high oil binding capacity makes it suitable to impart viscosity and adhesiveness to the food systems. From a rheological standpoint, the reduction of fats destabilizes the emulsions, thus, the application of grapefruit albedo could support the three dimensional network of proteins and stabilize the system. It should be acknowledged that the physicochemical properties of citrus flavedo and albedo are most likely due to their high content of fiber.

Besides useful functionalities, citrus flavedo and albedo provide great amounts of phenolic compounds. The use of enzymes resulted an effective method to augment the extraction of phenolics, also from industrial citrus waste. Pectinase and cellulase maximized the yields of phenolic compounds, prompted their partial conversion into aglycones, and the extraction of phenolic acids bound to cell wall polymers. In particular, the treatment with pectinase was the most effective in producing and/or releasing aglycones from albedo, e.g., luteolin, dihydrokaempferol, and hesperetin. Results from this investigation demonstrate that processes of enzymatic hydrolysis of citrus peel tissues could be used for nutraceutical and pharmaceutical purposes.

Funding Funding was provided by Symrise AG, Holzminden, Germany.

\section{References}

1. N. Mahato, K. Sharma, M. Sinha, M.H. Cho, Citrus waste derived nutra-/pharmaceuticals for health benefits: current trends and future perspectives. J. Funct. Foods 40, 307-316 (2018)

2. S.N. Joglekar, P.D. Pathak, S.A. Mandavgane, B.D. Kulkarni, Process of fruit peel waste biorefinery: a case study of citrus waste 
biorefinery, its environmental impacts and recommendations. Environ. Sci. Pollut. Res. 26, 34713-34722 (2019)

3. F. Teixeira, B.A. dos Santos, G. Nunes et al., Addition of orange peel in orange jam: evaluation of sensory, physicochemical, and nutritional characteristics. Molecules 25, 1670 (2020)

4. FAOSTAT. (2020) In: The Food and Agriculture Organization of the United Nations. http://www.fao.org/faostat/en/\#data/QC. Accessed 13 May 2020

5. J. Ke, G. Jiang, G. Shen et al., Optimization, characterization and rheological behavior study of pectin extracted from chayote (Sechium edule) using ultrasound assisted method. Int. J. Biol. Macromol. 147, 688-698 (2020)

6. S.S. Hosseini, F. Khodaiyan, M. Kazemi, Z. Najari, Optimization and characterization of pectin extracted from sour orange peel by ultrasound assisted method. Int. J. Biol. Macromol. 125, 621-629 (2019)

7. P. Chavan, A.K. Singh, G. Kaur, Recent progress in the utilization of industrial waste and by-products of citrus fruits: a review. J. Food Process. Eng. 41, e12895 (2018)

8. P. Putnik, D.B. Kovacevic, A.R. Jambrak et al., Innovative "green" and novel strategies for the extraction of bioactive added value compounds from citrus wastes-a review. Molecules 22, 680 (2017)

9. V. Sicari, T.M. Pellicano, A.M. Giuffre et al., Physical chemical properties and antioxidant capacities of grapefruit juice (Citrus paradisi) extracted from two different varieties. Int. Food Res. J. 25, 1978-1984 (2018)

10. V. Sicari, T.M. Pellicano, A.M. Giuffre et al., Bioactive compounds and antioxidant activity of citrus juices produced from varieties cultivated in Calabria. J. Food Meas. Charact. 10, 773780 (2016)

11. S. Multari, C. Licciardello, M. Caruso, S. Martens, Monitoring the changes in phenolic compounds and carotenoids occurring during fruit development in the tissues of four citrus fruits. Food Res. Int. 134, 109228 (2020)

12. N. M'hiri, I. Ioannou, M. Ghoul, N.M. Boudhrioua, Phytochemical characteristics of citrus peel and effect of conventional and nonconventional processing on phenolic compounds: a review. Food Rev. Int. 33, 587-619 (2017)

13. A.R. Ruviaro, P. de Menezes Barbosa, G.A. Macedo, Enzymeassisted biotransformation increases hesperetin content in citrus juice by-products. Food Res. Int. 124, 213-221 (2019)

14. J. Nishad, S. Saha, C. Kaur, Enzyme- and ultrasound-assisted extractions of polyphenols from Citrus sinensis (cv. Malta) peel: a comparative study. J. Food Process. Preserv. 43, 14046 (2019)

15. Y. Zhang, J. Qi, W. Zeng et al., Properties of dietary fiber from citrus obtained through alkaline hydrogen peroxide treatment and homogenization treatment. Food Chem. 311, 125873 (2020)

16. O.O. Olukomaiya, O.Q. Adiamo, W.C. Fernando et al., Effect of solid-state fermentation on proximate composition, anti-nutritional factor, microbiological and functional properties of lupin flour. Food Chem. 315, 126238 (2020)

17. S. Multari, I. Carafa, L. Barp et al., Effects of Lactobacillus spp. on the phytochemical composition of juices from two varieties of Citrus sinensis L. Osbeck: "Tarocco" and "Washington navel." LWT Food Sci. Technol. 125, 109205 (2020)

18. S. Schalow, M. Baloufaud, T. Cottancin et al., Orange pulp and peel fibres: pectin-rich by-products from citrus processing for water binding and gelling in foods. Eur. Food Res. Technol. 244, 235-244 (2018)
19. U.R. Ramasamy, H. Gruppen, M.A. Kabel, Water-holding capacity of soluble and insoluble polysaccharides in pressed potato fibre. Ind. Crop Prod. 64, 242-250 (2015)

20. M.C. Lopez-Marcos, C. Bailina, M. Viuda-Martos et al., Properties of dietary fibers from agroindustrial coproducts as source for fiber-enriched foods. Food Bioprocess. Technol. 8, 2400-2408 (2015)

21. Y. Liu, A. Liu, S.A. Ibrahim et al., Isolation and characterization of microcrystalline cellulose from pomelo peel. Int. J. Biol. Macromol. 111, 717-721 (2018)

22. F. Ye, B. Tao, J. Liu et al., Effect of micronization on the physicochemical properties of insoluble dietary fiber from citrus (Citrus junos Sieb. ex Tanaka) pomace. Food Sci. Technol. Int. 22, 246-255 (2016)

23. M. Ben Zid, C. Dhuique-Mayer, S. Bellagha et al., Effects of blanching on flavanones and microstructure of Citrus aurantium peels. Food Bioprocess. Technol. 8, 2246-2255 (2015)

24. M. Ben Zid, C. Dhuique-Mayer, M. Lartaud et al., Effects of osmotic treatments on modulating bitter flavanones glycosides contents and microstructure of Citrus aurantium peels. Food Bioprocess. Technol. 8, 2461-2469 (2015)

25. S. Giovanelli, D. Ciccarelli, G. Giusti et al., Comparative assessment of volatiles in juices and essential oils from minor Citrus fruits (Rutaceae). Flavour Frag. J. 35, 639-652 (2020)

26. M. Miura, M. Nogami, M. Sakai et al., Rapid LC-MS/ MS determination of hesperidin in fermented tea prepared from unripe satsuma mandarin (Citrus unshiu) fruits and third- crop green tea (Camellia sinensis) leaves. Anal. Sci. 36, 1243-1249 (2020)

27. C. Guo, Y. Shan, Z. Yang et al., Chemical composition, antioxidant, antibacterial, and tyrosinase inhibition activity of extracts from Newhall navel orange (Citrus sinensis Osbeck cv. Newhall) peel. J. Sci. Food Agric. 100, 2664-2674 (2020)

28. N. Zarate-Vilet, C. Wisniewski, E. Gue, M. Delalonde, Towards a better identification of naringin and narirutin dispersion state in grapefruit peel press liquor. Chem. Eng. Res. Des. 159, 205-214 (2020)

29. C. Le Bourvellec, C.M.G.C. Renard, Interactions between polyphenols and macromolecules: quantification methods and mechanisms. Crit. Rev. Food Sci. Nutr. 52, 213-248 (2012)

30. N. Bordenave, B.R. Hamaker, M.G. Ferruzzi, Nature and consequences of non-covalent interactions between flavonoids and macronutrients in foods. Food Funct. 5, 18-34 (2014)

31. S. Multari, A. Marsol-Vall, M. Keskitalo et al., Effects of different drying temperatures on the content of phenolic compounds and carotenoids in quinoa seeds (Chenopodium quinoa) from Finland. J. Food Compos. Anal. 72, 75-82 (2018)

32. T. Lau, N. Harbourne, M.J. Oruna-Concha, Optimization of enzyme-assisted extraction of ferulic acid from sweet corn cob by response surface methodology. J. Sci. Food Agric. 100, 14791485 (2020)

Publisher's Note Springer Nature remains neutral with regard to jurisdictional claims in published maps and institutional affiliations. 


\section{Affiliations}

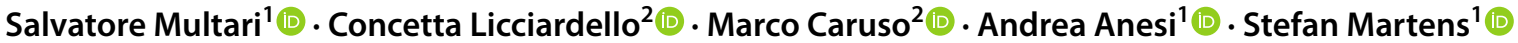

$\bowtie$ Salvatore Multari

s.multari@hotmail.it

1 Research and Innovation Centre, Fondazione Edmund Mach, San Michele All' Adige, TN, Italy
2 CREA, Research Centre for Olive, Fruit and Citrus Crops, Acireale, Italy 